Communications in Physics, Vol. 30, No. 2 (2020), pp. 151-159

DOI:10.15625/0868-3166/30/2/14857

\title{
SIMULATION STUDY OF MID-INFRARED SUPERCONTINUUM GENERATION AT NORMAL DISPERSION REGIME IN CHALCOGENIDE SUSPENDED-CORE FIBER INFILTRATED WITH WATER
}

\author{
BIEN CHU VAN ${ }^{1}$, MAI DANG NGOC ${ }^{1}$, VAN CAO LONG ${ }^{2}$, \\ HOANG NGUYEN TUAN ${ }^{3}$ AND 'HIEU LE VAN ${ }^{1, \dagger}$ \\ ${ }^{1}$ Department of Physics, Hong Duc University, 565 Quang Trung Street, Thanh Hoa City, Vietnam \\ ${ }^{2}$ Institute of Physics, University of Zielona Góra, Prof. Szafrana 4a, 65-516 Zielona Góra, Poland \\ ${ }^{3}$ Military College of Special Forces, Chuong My District, Hanoi City, Vietnam \\ ${ }^{\dagger} E$-mail: levanhieu@hdu.edu.vn
}

Received 27 February 2020

Accepted for publication 19 March 2020

Published 12 May 2020

\begin{abstract}
We report simulation results of supercontinuum generation in the suspended-core optical fibers made of chalcogenide $\left(\mathrm{As}_{2} \mathrm{~S}_{3}\right)$ infiltrated with water at mid-infrared wavelength range. Applying water-hole instead of the air-hole in fibers allows improving the dispersion characteristics, hence, contributing to supercontinuum generations. As a result, the broadband supercontinuum generation ranging from $1177 \mathrm{~nm}$ to $2629 \mathrm{~nm}$ was achieved in a $10 \mathrm{~cm}$ fiber by utilizing very low input pulse energy of $0.01 \mathrm{~nJ}$ and pulse duration of $100 \mathrm{fs}$ at $1920 \mathrm{~nm}$ wavelength.
\end{abstract}

Keywords: suspended-core; normal dispersion; supercontinuum generation.

Classification numbers: 42.81.Gs; 63.20.D; 42.65.Jx; 88.60.np.

(C)2020 Vietnam Academy of Science and Technology 


\section{INTRODUCTION}

Based on the geometrical structure, the microstructures optical fibers (MOFs) can be divided into two groups: hollow-core (HC) and solid core fibers [1-8]. In the case of HC-MOFs, with a refractive index in the cladding higher than that in the core, light will be trapped and guided inside the hollow core due to photonics band gap with highly transmitted power, which is very interesting for optical spectroscopy [1]. However, a significant drawback of the HC-MOFs is a limited spectral range. Thus, the HC-MOFs are very well suited for a narrow band chemical sensing [2]. On the contrary, a broad-band sensing is possible with the solid core-MOFs [6-8]. For this fiber, light is guided through the supercontinuum (SC) based on total internal reflection and only a little power is transmitted via the holes. Suspended-core-fibers (SCFs) are a particular example for the solid core-MOFs. A suspended-core fiber usually consists of a small core suspended between three submicron-thin bridges made from glasses $[9,10]$. For particular micro-structured designs of fibers, one can obtain ultra-confinement of modes. Modulating the size and shape of fiber cores could introduce high birefringence or nonlinearities which lead to the presence of SC phenomena and promise applications in biomedical or chemical sensing $[6,11]$.

SC laser sources in MOFs have been an attractive topic thanks to their numerous potential applications in different research areas, for instance, among others, fiber sensing [12], optical coherence tomography [13], frequency metrology [14], and spectroscopy [15]. SC generation is a complex process of spectral broadening where the output spectrum becomes much wider than the input spectrum when an ultrashort optical pulse passes through a nonlinear medium. For optical waveguide, spectral broadening phenomena in the anomalous dispersion region is physically caused by few factors named soliton fission and dispersive waves [12] when we use femtosecond or picosecond pulse to pump sources having wavelength near the zero-dispersion wavelength (ZDW). Meanwhile, the SC generation taking place in the normal dispersion region is due to effects of optical wave breaking (OWB) involving four-wave mixing (FWM) and self-phase modulation (SPM) $[16,17]$. Although, the phenomena in the latter case usually have low bandwidth and much higher energy requirements for efficient excitation but exhibits SC results high stability with low noise and a better pulse-to-pulse temporal coherence [18].

Over the last decades, there has been more attention of mid-infrared (mid-IR) spectral region since it can contribute to study about chemical components of organic compounds by their distinctive spectral fingerprints [16]. Indeed, the mid-IR light plays an important role for various applications including gas sensing [6], medical diagnostics [17], especially food quality control [19]. In particular, using the fused silica fiber the broadband SC in regions of the visible and near-IR can be resulted $[6,12]$. However, there are several limitations of using the generating medium of silica fibers. The limit of the intrinsic transmission window of fused silica produces SC spectral evolution in mid-IR. Therefore, the development of SC generation sources from nonsilica, highly nonlinear material becomes significant. In fact, several non-silica materials such as tellurite [20], heavy metal oxide [21], and chalcogenide based materials [22] have been designed for mid-IR fiber SC sources. In addition, using photonic crystal fibers (PCFs) by filling the air holes with various liquids was considered [23-25]. The application of liquids allows modifying fiber dispersion properties with an unnecessary change of geometrical parameters. That dispersion characteristic curvature of fibers could further be altered dramatically. 
In this paper, we make a report on numerical study of mid-IRSC generation by pumping at normal dispersion regime of chalcogenide suspended-core fiber infiltrated with water. Owning wider transmission window together with higher nonlinear properties in mid-IR region [26], $\mathrm{As}_{2} \mathrm{~S}_{3}$ glass shows impressive advantages in comparison with other mentioned materials. In addition, water is the most typical solvent presented in almost compounds of bimolecular and organic. Furthermore, it is possible to create a nonlinear medium for SC formation by utilizing water doped with bioluminescent.

The work is organized in three main steps. The first one is to optimize the dispersion properties via modifying geometrical parameters. In particular, diameter of air-hole is determined in order to obtain the dispersion flatness in the normal region, as well as the wavelength for maximum value of the fiber dispersion curve having to be the closest one to the pumping wavelength. Then, SC generation will be demonstrated for optimal fiber structure by solving generalized nonlinear Schrödinger equation (GNLSE). In the final step, the discussions about the advantages of the proposed fiber are presented.

\section{MODELING OF THE SUSPENDED-CORE OPTICAL FIBER INFILTRATED WITH WATER}

The developed fiber was investigated numerically basing on scanning electron microscopy (SEM) photos. We consider the SCF made of chalcogenide glass consisting of three equivalent holes (Fig. 1a), in which the holes are filled with water. The total diameter of air-hole structure D is $32.14 \mu \mathrm{m}$, diameter of the core area $2 r_{c}$ is roughly $1.64 \mu \mathrm{m}$, radius of a hole $R_{\text {hole }}$ is $15.23 \mu \mathrm{m}$, and the struts $t_{c}$ are just $0.25 \mu \mathrm{m}$ thick (\# F1).

For modeling, we used the MODEL Solution software to calculation of the modal properties of SCF [27], in which all imperfection of the real fiber structures was taken into account. The geometrical parameters of the SCF are as in Table 1.

Table 1. Geometrical parameters of fabricated SCF.

\begin{tabular}{ll}
\hline Parameters & \# F1 \\
\hline Core diameter $2 \mathrm{r}_{c}[\mu \mathrm{m}]$ & 1.64 \\
Thickness of glass bridges $\mathrm{t}_{c}[\mu \mathrm{m}]$ & 0.25 \\
Radius of holes $\mathrm{R}_{\text {hole }}[\mu \mathrm{m}]$ & 15.23 \\
Total diameter of air-hole structure $\mathrm{D}[\mu \mathrm{m}]$ & 32.14 \\
\hline
\end{tabular}

The refractive index of the chalcogenide glass is given by following formula which was derived from the Sellmeier equation [28]:

$$
n(\lambda)=\sqrt{1+\frac{B_{1} \lambda^{2}}{\lambda^{2}-C_{1}}+\frac{B_{2} \lambda^{2}}{\lambda^{2}-C_{2}}+\frac{B_{3} \lambda^{2}}{\lambda^{2}-C_{3}}}
$$

where $B_{1}=0.6694226, B_{2}=0.4345839, B_{3}=0.8716947$ and $C_{1}=0.0044801 \mu \mathrm{m}^{2}, C_{2}=0.013285$ $\mu \mathrm{m}^{2}, C_{3}=95.341482 \mu \mathrm{m}^{2}$ are Sellmeier coefficients and $\lambda$ is the operating wavelength $(\mu \mathrm{m})$. 

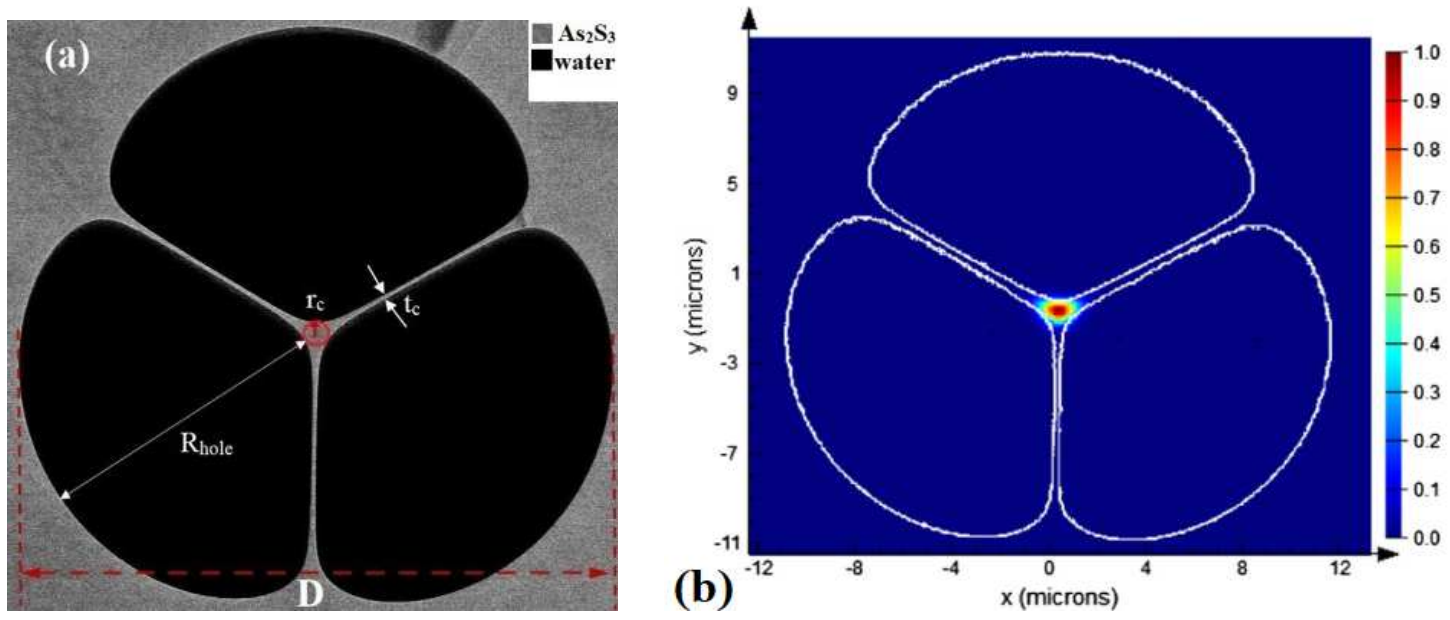

Fig. 1. (a) Cross section of SCF, (b) numerically calculated intensity distribution of the fundamental mode of SCF \# F5 at $1920 \mathrm{~nm}$.

Cauchy formula with temperature dependent coefficients has to be used for the case of water [29]:

$$
n_{w}(\lambda, t)=A(t)+\frac{B(t)}{\lambda^{2}}+\frac{C(t)}{\lambda^{4}}+\frac{D(t)}{\lambda^{6}}
$$

where $\lambda$ is wavelength $(\mathrm{nm}) ; t$ is the temperature $\left({ }^{\circ} \mathrm{C}\right)$ and Cauchy coefficients $A(t), B(t), C(t)$, $D(t)$ are presented as functions of temperature:

$$
\begin{aligned}
& A(t)=1.3208-1.2325 .10^{-5} t-1.8674 .10^{-6} t^{2}+5.0233 .10^{-9} t^{3} \\
& B(t)=5208.2413-0.5179 t-2.284 .10^{-2} t^{2}+6.9608 .10^{-5} t^{3} \\
& C(t)=-2.5551 .10^{8}-18341.336 t-917.2319 t^{2}+2.7729 t^{3} \\
& D(t)=9.3495+1.7855 .10^{-3} t+3.6733 .10^{-5} t^{2}-1.2932 .10^{-7} t^{3}
\end{aligned}
$$

Furthermore, we only consider for the fundamental mode and we assume that increasing temperature makes the refractive index contrast between the glass core and water in the fiber holes increased.

\section{OPTIMIZATION OF ALL NORMAL DISPERSION PROPERTIES FOR SUSPENDED-CORE OPTICAL FIBER WITH VARIOUS SIZES}

This part is for the discussion about the optimization of SCF structure, in which a number of simulations for SCFs having different geometry parameters were conducted. The optimization criteria aimed at the generation of SC in all normal dispersion regimes with pumping at $1.92 \mu \mathrm{m}$. We assumed that it is possible to modify the total structure diameter and also other parameters within a reasonable range. Thus, in order to have a wider overlook, we implemented simulations using sets of rescaled parameters from the ones for fabricated SCF \# F1. Table 2 presents the proposed geometrical parameters for our numerical calculations. 
Figure 2 depicts the dispersion characteristics for the fundamental mode in the range from $0.5 \mu \mathrm{m}$ to $5.0 \mu \mathrm{m}$. As a result, when the core diameter increases the characteristics curve of dispersion becomes flatter, and the localization of the ZDW is shifted toward a longer wavelength. For fiber \# F1 having a $1.64 \mu \mathrm{m}$ core diameter, the first ZDW is at $1.955 \mu \mathrm{m}$, while for fiber \# F6 with the core diameter of $0.984 \mu \mathrm{m}$, the first ZDW is of $1.82 \mu \mathrm{m}$. Moreover, for fiber \# F8 with the core diameter of $1.968 \mu \mathrm{m}$, the first ZDW can be achieved at $2.055 \mu \mathrm{m}$.

Table 2. Geometry parameters of designed SCFs made of $\mathrm{As}_{2} \mathrm{~S}_{3}$.

\begin{tabular}{llllll}
\hline Fiber & $\mathrm{D}(\mu \mathrm{m})$ & $\mathrm{r}_{c}(\mu \mathrm{m})$ & $\mathrm{t}_{c}(\mu \mathrm{m})$ & $\mathrm{R}_{\text {hole }}(\mu \mathrm{m})$ & Scale $(\%)$ \\
\hline \# 2 & 12.856 & 0.328 & 0.1 & 6.092 & 40 \\
$\# 33$ & 14.463 & 0.369 & 0.1125 & 6.8535 & 45 \\
$\# 4$ & 16.07 & 0.41 & 0.125 & 7.615 & 50 \\
$\# 5$ & 17.677 & 0.451 & 0.1375 & 8.3765 & 55 \\
$\#$ 6 & 19.284 & 0.492 & 0.15 & 9.138 & 60 \\
$\# 7$ & 25.712 & 0.656 & 0.2 & 12.184 & 80 \\
$\# 1$ & 32.14 & 0.82 & 0.25 & 15.23 & 100 \\
$\# 8$ & 38.568 & 0.984 & 0.3 & 18.276 & 120 \\
$\# 9$ & 44.996 & 1.148 & 0.35 & 20.122 & 140 \\
\hline
\end{tabular}

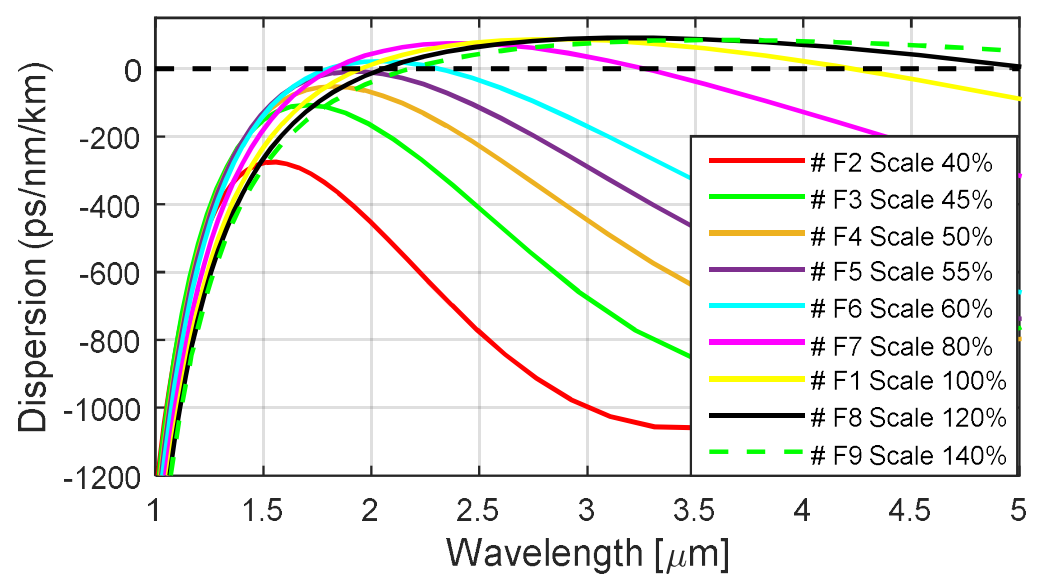

Fig. 2. Dispersion curves of modeled SCF infiltrated with water for various core diameters.

On the basis of initial numerical investigations, we selected fiber \# F5. This fiber has optimum dispersion characteristics for SC generation since this fiber has all normal dispersion as 
well as the wavelength located at maximum point on the fiber dispersion characteristics being the closest wavelength to the pumping one

In addition, the optimal fiber structure without water is characterized by anomalous dispersion where the first ZDW is equal to $1.5525 \mu \mathrm{m}$, the second ZDW equal to $2.6245 \mu \mathrm{m}$, and the dispersion at the $1.92 \mu \mathrm{m}$ wavelength equals $113.9 \mathrm{ps} / \mathrm{nm} / \mathrm{km}$ (Fig. 3).

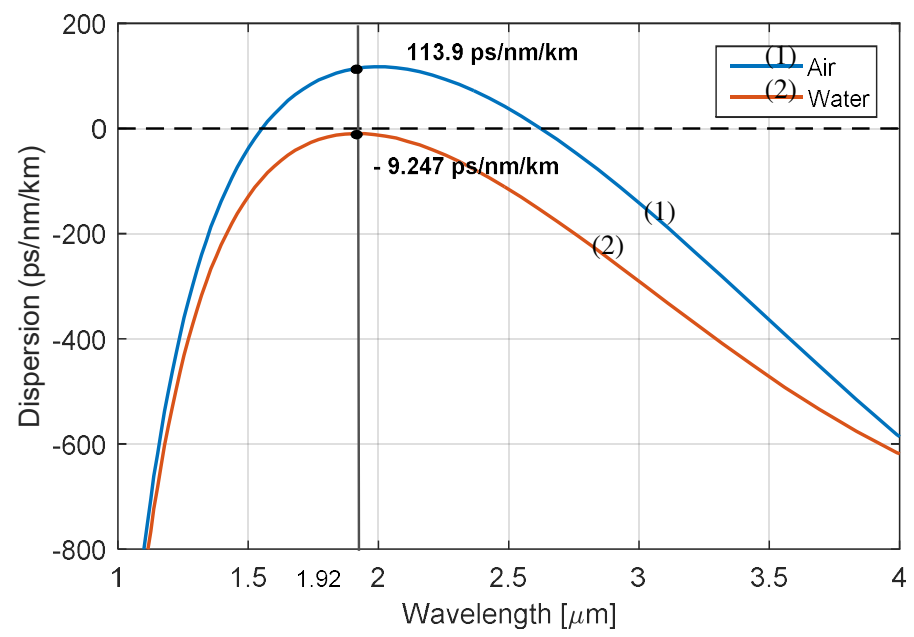

Fig. 3. Numerical calculations of dispersion characteristics in fiber \# F5 with air holes and infiltrated with water.

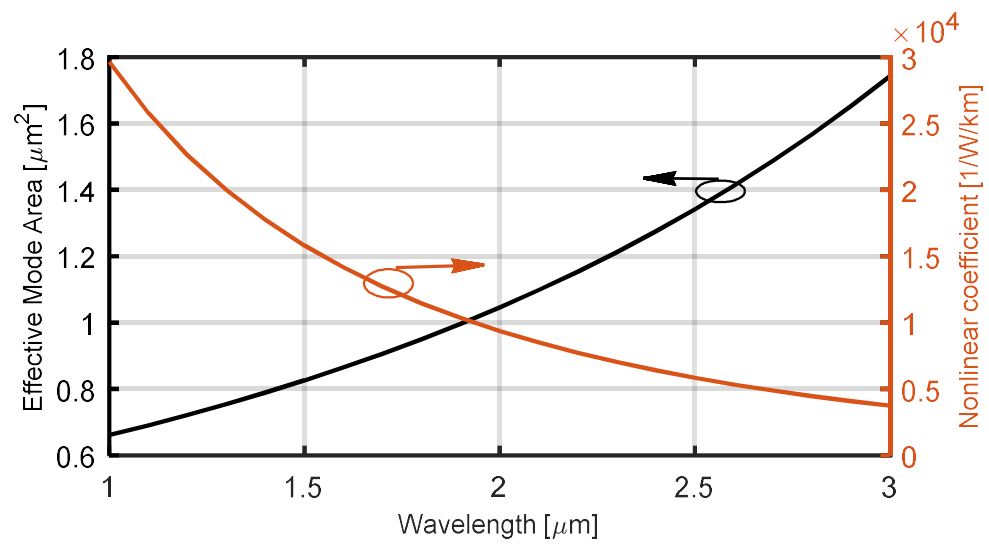

Fig. 4. Calculations of effective mode area and the nonlinear coefficient of SCF infiltrated with water.

The effective mode area and nonlinear coefficients of the optimal structure are presented in Fig. 4. The fundamental mode of our structure is very well confined in the core, while the modal area of the fundamental mode increases linearly with the wavelength. For the wavelength 
of $1.0 \mu \mathrm{m}$ the modal area equals $0.66125 \mu \mathrm{m}^{2}$, while for the wavelength of $3.0 \mu \mathrm{m}$, the modal area equals $1.74381 \mu \mathrm{m}^{2}$. So the mode area is not changed much within the wavelength range.

\section{SUPERCONTINUUM GENERATION IN ALL NORMAL DISPERSION OF A SUSPENDED-CORE OPTICAL FIBER INFILTRATED WITH WATER}

In the next step, we were to calculate the SC generated in optimized SCF structure for the fundamental mode. For this purpose, the generalized nonlinear Schrödinger equation (GNLSE) was solved by using the split-step Fourier method [15]:

$\frac{\partial A}{\partial z}=-\frac{\alpha}{2} A+\sum_{n \geq 2} \beta_{n} \frac{i^{n+1}}{n !} \frac{\partial^{n}}{\partial T^{n}} A+i \gamma \frac{1}{\omega_{0}}\left(1+\frac{\partial}{\partial T}\right)\left[\left(1-f_{R}\right)|A|^{2} A+f_{R} A \int_{0}^{\infty} h_{R}(t)|A(z, T-t)|^{2} d t\right]$

where $A=A(z, t)$ is the complex amplitude of the optical field, $\alpha$ is the total loss in the SCF, $\beta_{n}$ is the dispersion coefficients associated with the Taylor series expansion, the nonlinear coefficient $\gamma$ is defined by Eq. (4), $\lambda_{c}$ is the central wavelength, $f_{R}$ is the Raman fraction response to nonlinear polarization, $h_{R}(t)$ represents the Raman response function which is given in Ref. [23]: $h_{R}(t)=$ $\left(\tau_{1}^{2}+\tau_{2}^{2}\right) \tau_{1}^{-1} \tau_{2}^{-2} \exp \left(-t / \tau_{2}\right) \sin \left(-t / \tau_{1}\right)$.

The analysis was simulated with the following parameters: the fiber length $10 \mathrm{~cm}$, the Gaussian-shape pulse of duration $100 \mathrm{fs}$, and the Raman fraction $f_{R}=0.031, \tau_{1}=15.2 \mathrm{fs}, \tau_{2}=$ 230.5 fs [26], the nonlinear refractive index of $\mathrm{As}_{2} \mathrm{~S}_{3} \quad n_{2}=1.1 \times 10^{-17}\left(\mathrm{~m}^{2} / \mathrm{W}\right)$ [30], and at wavelength of $1.92 \mu \mathrm{m}$.
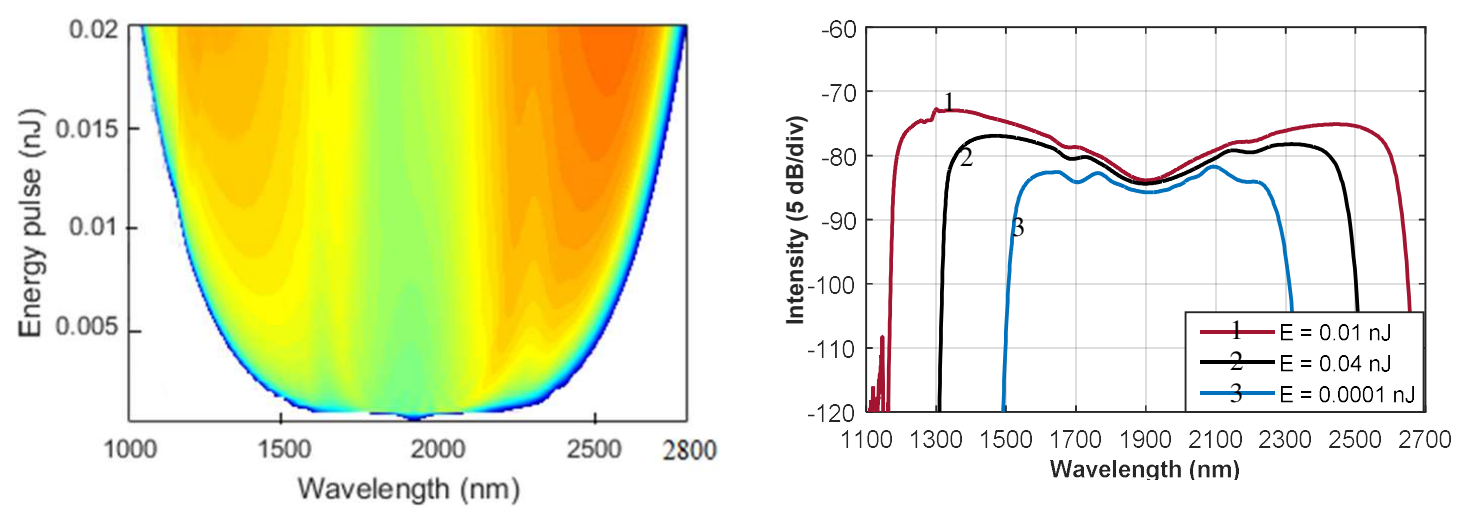

Fig. 5. Spectral intensity of SCF with different pulse energies.

The performance of spectrum broadening at the fiber length of $10 \mathrm{~cm}$ for various input pulse energies is presented in Fig. 5. In the case of input pulse energy being below $0.04 \mathrm{~nJ}$, the initial widening of the spectrum is mainly from SPM. The OWB begins to show up when the input pulse energy is higher than $0.04 \mathrm{~nJ}$. For input pulse energy of $0.01 \mathrm{~nJ}$, the SCF can be expected with bandwidth of $1451 \mathrm{~nm}$ around the pumping wavelength in the range $1177-2629 \mathrm{~nm}$ of wavelengths. Further increase in the spectral width can be expected if we increase input pulse energy. 

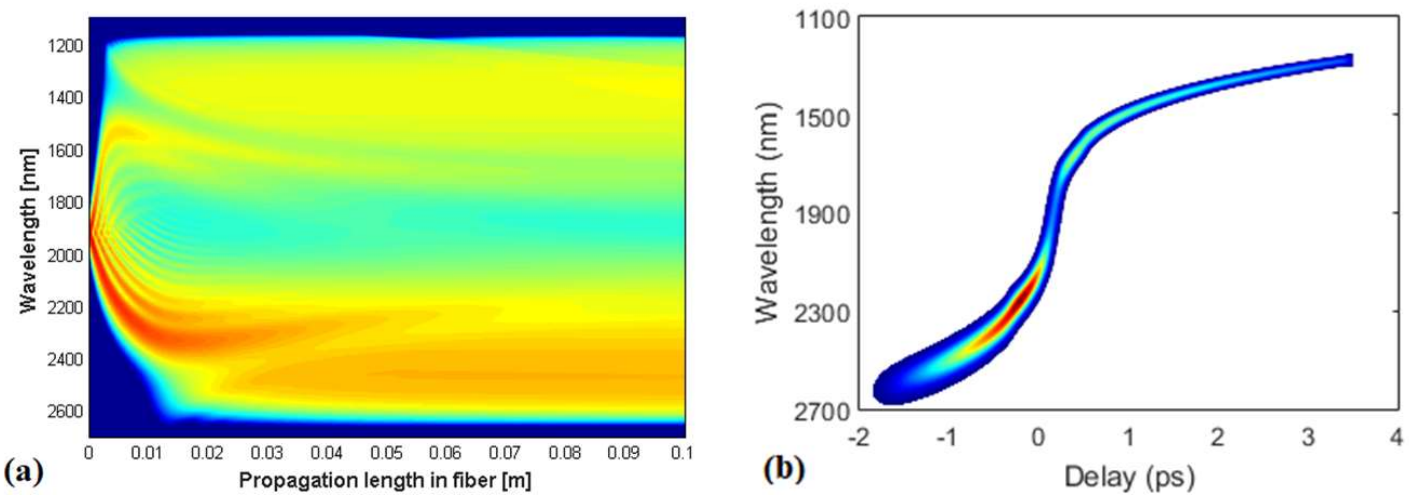

Fig. 6. Numerical calculations of the spectral (a) and temporal evolution (b) of the pulse along the fiber in the SCF infiltrated with water.

Figure 6 presents the spectral and temporal evolution of the pulse along the propagation distance for the water-filled-suspended-core fiber with input pulse energy $0.01 \mathrm{~nJ}$. In this case, the location of pump wavelength is in the normal dispersion region, the nonlinear process begins with SPM and the optical pulse is broadened symmetrically around the pump wavelength. Next, we observe a further widening of the spectrum because of optical wave breaking. After a few centimeters of propagation length, the spectrum broadening process has ended and the spectrum only becomes smoother due to degenerated FWM mixing effect.

\section{CONCLUSION}

In this work, we have optimized the geometrical parameters of a chalcogenide suspendedcore fiber infiltrated with water to obtain all-normal dispersion characteristic. Simulations that were conducted to obtain a flat dispersion and the generation of SC in the whole normal dispersion region with pumping at $1.92 \mu \mathrm{m}$ allowed the optimization of the SCF structure: total diameter of air-hole structure $\mathrm{D}$ is of $17.677 \mu \mathrm{m}$, diameter of the core area $2 \mathrm{r}_{c}$ is $0.902 \mu \mathrm{m}$, radius of holes $\mathrm{R}_{\text {hole }}$ is $8.3765 \mu \mathrm{m}$, and the struts $t_{c}$ are just $0.1375 \mu \mathrm{m}$ thick (\# F5). Our numerically calculated results demonstrate that in the optimal SCF infiltrated with water, the SC spectral bandwidth broadens from 1177 to $2629 \mathrm{~nm}$ when the input pulse had pump wavelength at $1920 \mathrm{~nm}$, pulse duration of $100 \mathrm{fs}$ and $0.01 \mathrm{~nJ}$ input pulse energy. Owning high nonlinearity of chalcogenide, octave-spanning SC can be obtained in a short length of the fiber (shorter than $2 \mathrm{~cm}$ ). The use of short fiber, as well as, short input pulse allowed suppressing the effect of polarization noise, laser noise. Thus we can use the chalcogenide suspended-core fiber infiltrated with water to generate $\mathrm{SC}$ in mid-IR, which has a significant role in biological imaging and optical coherent tomography $[13,17]$.

\section{ACKNOWLEDGMENT}

We would like to express our gratitude to Prof. Ryszard Buczyñski and Prof. Rafal Kasztelanic for useful discussions. 


\section{REFERENCES}

[1] K. Gauthron, J-S. Lauret, L. Doyennette, G. Lanty, A. Al Choueiry, S.J. Zhang, A. Brehier, L. Largeau, O. Mauguin, J. Bloch, and E. Deleporte, Opt. Express 18 (2010) 1094.

[2] Y. Cho, B. Park, J. Oh, M. Seo, K. Lee, C. Kim, T. Lee, D. H. Woo, S. Lee, H. M. Kim, H.J. Lee, K. Oh, D. I. Yeom, S. R. Dugasani, S. H. Park, and J. H. Kim, Opt. Express 23 (10) (2015) 1094.

[3] J. C. Knight, J. Broeng, T. A. Birks, P. St. J. Russell, Science 282 (5393) (1998)1476-1478.

[4] R. F. Cregan, B. J. Mangan, C. Knight, T. A. Birks, P. St. J. Russell, P. J. Roberts, and D. C. Allan, Science 285 (5433) (1999) 1537.

[5] T. T. Alkeskjold, PhD. Thesis, Department of Communication, Optics \& Materials, Technical University of Denmark (2005).

[6] J. M. Dudley, G. Genty, and S. Coen, Rev. Mod. Phys. 78 (2006) 1135

[7] W. Jin, J. Ju, H. L. Ho, Y. L. Hoo, A. Zhang, Front. Optoelectron. 6 (2013) 3.

[8] G. Stepniewski, R. Kasztelanic, D. Pysz, R. Stepien, M. Klimczak, and R. Buczynski, Optical Materials Express 6(8) (2016) 2159-3930.

[9] L. Dong, B. K. Thomas, and L. Fu, Opt. Express 16 (2008) 16423.

[10] A. Yu. Chamorovskiy, and S. A. Nikitov, J. Commun. Technol. Electron. 58 (2013) 879.

[11] G. P. Agrawal, Nonlinear Fiber Optics, 4th edition, New York, Academic Press (2007)

[12] T. M. Monro, W. Belardi, K. Furusawa, J. C. Baggett, N. G. R. Broderick, and D. J. Richardson, Meas. Sci. Technol. 12 (2001) 854.

[13] S. T. Cundiff and J. Ye, Rev. Mod. Phys. 75 (2003) 325.

[14] M. J. Thorpe, D. D. Hudson, K. D. Moll, J. Lasri and J. Ye, Opt. Lett. 32 (2007) 307.

[15] G. P. Agrawal, Nonlinear Fiber Optics 5th edn, 2013 (Oxford: Academic Press).

[16] A. Schliesser, N. Picqué and T. W. Hänsch, Nat. Photonics 6 (2012) 440-449.

[17] B. Guo, Y. Wang, C. Peng, H. L. Zhang, G. P. Luo, H. Q. Le, C. Gmachl, D. L. Sivco, M. L. Peabody and A.Y. Cho, Opt. Express 12 (2014) 208.

[18] G. Stepniewski, M. Klimczak, H. Bookey, B. Siwicki, D. Pysz, R. Stepien, A. K. Kar, A. J. Waddie, M. R. Taghizadeh and R. Buczynski, Laser Phys. Lett. 11 (2014) 055103.

[19] R. Wilson and H. Tapp, TRAC-Trend. Anal. Chem. 18 (1999) 85.

[20] P. Domachuk, N. A. Wolchover, M. Cronin-Golomb, A. Wang, A. K. George, C. M. B. Cordeiro, J. C. Knight and F. G. Omenetto, Opt. Express 16 (2008) 7161.

[21] C. Xia et al., IEEE J. Sel. Top. Quant. 15 (2009) 422.

[22] R. Buczynski, H. T. Bookey, D. Pysz, R. Stepien, I. Kujawa, J. E. McCarthy, A. J. Waddie, A. K. Kar, and M. R. Taghizadeh, Laser Phys. Lett. 7 (2010) 666.

[23] H. Le Van, V. C. Long, H. T. Nguyen, A. M. Nguyen, R. Buczynski R. Kasztelanic, Laser Physics 28 (2018) 1054-660X.

[24] V. T. Hoang, R. Kasztelanic, A. Filipkowski, G. Stêpniewski, D. Pysz, M. Klimczak, S. Ertman, V. C. Long, T.R. Woliñski, M. Trippenbach, K. D. Xuan, M. Śmietana, and R. Buczyñski, Opt. Mater. Express 9 (2019) 2159.

[25] L. C. Van, V. T. Hoang, V. C. Long, K. Borzycki, K. D. Xuan, V. T. Quoc, M. Trippenbach, R. Buczyñski and J. Pniewski, Laser Phys. 30 (2020) 1054.

[26] B. J. Eggleton, B. Luther-Davies, and K. Richardson, Nat. Photonics 5 (2011) 141.

[27] Lumerical Solutions, Inc., http://www.lumerical.com.

[28] Web page: Refractive Index Info: https://refractiveindex.info.

[29] N. P. Barnes and M. S. Piltch, Opt. Soc. A 67 (1977) 628.

[30] M. E. Amraoui, J. Fatome, J. C. Jules, B. Kibler, G. Gadret, C. Fortier, F. Smektala, I. Skripatchev, C.F. Polacchini, Y. Messaddeq, J. Troles, L. Brilland, M. Szpulak, and G. Renversez, Opt. Express 18 (2010) 4547. 